TRANSACTIONS OF THE

AMERICAN MATHEMATICAL SOCIETY

Volume 354, Number 1, Pages 265-274

S 0002-9947(01)02864-1

Article electronically published on August 20, 2001

\title{
ON NONLINEAR OSCILLATIONS IN A SUSPENSION BRIDGE SYSTEM
}

\author{
ZHONGHAI DING
}

\begin{abstract}
In this paper, we study nonlinear oscillations in a suspension bridge system governed by two coupled nonlinear partial differential equations. By applying the Leray-Schauder degree theory, it is proved that the suspension bridge system has at least two solutions, one is a near-equilibrium oscillation, and the other is a large amplitude oscillation.
\end{abstract}

\section{INTRODUCTION}

The suspension bridge is a common type of civil engineering structure. It is well known that suspension bridges may display certain oscillations under external aerodynamic forces. Under the action of a strong wind, for example, a narrow and very flexible suspension bridge can undergo dangerous oscillations [1]. Based upon the observation of the fundamental nonlinearity in suspension bridges that the stays connecting the supporting cables and the roadbed resist expansion, but do not resist compression, new models describing oscillations in suspension bridges have been developed recently by Lazer and McKenna in [10. The new models are described by systems of coupled nonlinear partial differential equations. The new study of suspension bridges initiated by Lazer and McKenna has produced many important and interesting results. Multiple large amplitude periodic oscillations have been found theoretically and numerically in the single Lazer-McKenna suspension bridge equation (see [3], [8]-10], [12] and references therein). However, there has been very little discussion on nonlinear periodic oscillations in suspension bridge systems of coupled nonlinear partial differential equations in the existing literature. In [2], Ahmed and Harbi investigated the asymptotic stability of a suspension bridge system governed by the coupled nonlinear beam and wave equations with nonlinear damping terms. The same system with linear damping terms has been studied also in [6] and [14], where the existence and uniqueness of near-equilibrium oscillation were studied. Except for the work mentioned above, the suspension bridge system governed by the coupled nonlinear beam and wave equations has not yet received in-depth study in the existing literature.

Received by the editors August 21, 2000 and, in revised form, April 3, 2001.

2000 Mathematics Subject Classification. Primary 35Q72, 47H11, 74H20.

Key words and phrases. Suspension bridge system, nonlinear oscillation, Leray-Schauder degree.

This research was supported in part by NSF Grant DMS 96-22910. 
In this paper, we study the following suspension bridge model proposed by Lazer and McKenna in [10]

$$
\left\{\begin{array}{l}
m_{c} u_{t t}-Q u_{x x}-K(w-u)^{+}=m_{c} g+\varepsilon h_{1}(x, t), \quad 0<x<L, \\
m_{b} w_{t t}+E I w_{x x x x}+K(w-u)^{+}=m_{b} g+\varepsilon h_{2}(x, t), \quad 0<x<L, \\
u(0, t)=u(L, t)=0, \\
w(0, t)=w(L, t)=0, \quad w_{x x}(0, t)=w_{x x}(L, t)=0,
\end{array}\right.
$$

which describes oscillations in a simplified suspension bridge configuration: the roadbed of length $L$ is modeled by a horizontal vibrating beam with both ends being simply supported; the supporting cable of length $L$ is modeled by a horizontal vibrating string with both ends being fixed; and the vertical stays connecting the roadbed to the supporting cable are modeled by one-sided springs which resist expansion but do not resist compression. In system (1.1), $u(x, t)$ and $w(x, t)$ denote the downward deflections of the cable and the roadbed, respectively; $(w-u)^{+}=$ $\max \{w-u, 0\} ; m_{c}$ and $m_{b}$ are the mass densities of the cable and the roadbed, respectively; $Q$ is the coefficient of cable tensile strength; $E I$ is the roadbed flexural rigidity; $K$ is the Hooke's constant of the stays; $h_{1}$ and $h_{2}$ represent the external periodic aerodynamic forces; and, $\varepsilon$ is a parameter. We are interested in periodic oscillations in (1.1), which are symmetric about $x=L / 2$,

$$
\begin{aligned}
& u(x, t+T)=u(x, t), \quad w(x, t+T)=w(x, t), \quad 0 \leq x \leq L, \\
& u(x, t)=u(L-x, t), \quad w(x, t)=w(L-x, t), \quad 0 \leq x \leq L,
\end{aligned}
$$

where $T$ is the period of periodic oscillations. By rescaling and translating $x$ and $t$, system (1.1) with (1.2) can be written in an equivalent form

$$
\left\{\begin{array}{l}
m_{c} u_{t t}-Q u_{x x}-K(w-u)^{+}=m_{c} g+\varepsilon h_{1}(x, t), \quad-\pi / 2<x<\pi / 2, \\
m_{b} w_{t t}+E I w_{x x x x}+K(w-u)^{+}=m_{b} g+\varepsilon h_{2}(x, t), \quad-\pi / 2<x<\pi / 2, \\
u(-\pi / 2, t)=u(\pi / 2, t)=0, \\
w(-\pi / 2, t)=w(\pi / 2, t)=0, \quad w_{x x}(-\pi / 2, t)=w_{x x}(\pi / 2, t)=0, \\
u(-x, t)=u(x, t), \quad w(-x, t)=w(x, t), \quad 0 \leq x \leq \pi / 2, \\
u(x, t+\pi)=u(x, t), \quad w(x, t+\pi)=w(x, t), \quad-\pi / 2 \leq x \leq \pi / 2,
\end{array}\right.
$$

where $h_{1}(x, t)$ and $h_{2}(x, t)$ are $\pi$-periodic functions in $t$.

We have studied in [4] nonlinear periodic oscillations of system (1.3) by assuming $h_{1}$ and $h_{2}$ being some special eigenfunctions of the beam and wave operators. By letting $h_{1}$ and $h_{2}$ be any $H^{2}$-functions and by applying the Mountain Pass Theorem, we have shown in [5] that system (1.3) has at least two periodic solutions.

By assuming $h_{1}$ and $h_{2}$ to be any $L^{2}$-functions, the objective of this paper is to study nonlinear periodic oscillations of system (1.3) by using the Leray-Schauder degree theory, which is motivated by an important paper [12 by McKenna and Walter who studied the single Lazer-McKenna suspension bridge equation by using the Leray-Schauder degree theory. It is proved in this paper that there exists a constant $\varepsilon_{0}>0$ such that system (1.3) has at least two periodic solutions if $|\varepsilon|<\varepsilon_{0}$ (see Theorem 2.2).

\section{NONLINEAR PERIODIC OSCILLATIONS}

To investigate the suspension bridge system (1.3), we assume throughout this paper that

$$
Q \leq m_{c}, \quad E I \leq m_{b},
$$


which hold naturally for suspension bridges in civil engineering applications. Define the wave operator $L_{1}$ by

$$
\left\{\begin{array}{l}
L_{1} u=m_{c} u_{t t}-Q u_{x x}, \\
u(-\pi / 2, t)=u(\pi / 2, t)=0, \\
u(x, t)=u(-x, t), \quad u(x, t+\pi)=u(x, t) .
\end{array}\right.
$$

Define the beam operator $L_{2}$ by

$$
\left\{\begin{array}{l}
L_{2} w=m_{b} w_{t t}+E I w_{x x x x}, \\
w(-\pi / 2, t)=w(\pi / 2, t)=0, \\
w_{x x}(-\pi / 2, t)=w_{x x}(\pi / 2, t)=0, \\
w(x, t)=w(-x, t), \quad w(x, t+\pi)=w(x, t) .
\end{array}\right.
$$

Denote by $\left\{\lambda_{m n}\right\}$ the eigenvalues of $L_{1}$ and by $\left\{\mu_{m n}\right\}$ the eigenvalues of $L_{2}$. Then it follows from a direct calculation that

$$
\begin{aligned}
& \lambda_{m n}=Q(2 n+1)^{2}-4 m_{c} m^{2}, \quad m, n=0,1,2, \cdots, \\
& \mu_{m n}=E I(2 n+1)^{4}-4 m_{b} m^{2}, \quad m, n=0,1,2, \cdots .
\end{aligned}
$$

The eigenfunctions of $L_{1}$ corresponding to eigenvalue $\lambda_{m n}$ are the same as that of $L_{2}$ corresponding to eigenvalue $\mu_{m n}$, which are given by

$$
\begin{aligned}
& \varphi_{0 n}(x, t)=\cos (2 n+1) x, \quad n \geq 0, \\
& \varphi_{m n}(x, t)=\cos (2 n+1) x \cos 2 m t, \quad m \geq 1, n \geq 0, \\
& \psi_{m n}(x, t)=\cos (2 n+1) x \sin 2 m t, \quad m \geq 1, n \geq 0 .
\end{aligned}
$$

Let $\Omega=(-\pi / 2, \pi / 2) \times(-\pi / 2, \pi / 2)$, and $H$ be the Hilbert space defined by

$$
H=\left\{u \in L^{2}(\Omega) \mid u(-x, t)=u(x, t)\right\} .
$$

It is easy to check that the set of eigenfunctions $\left\{\varphi_{m n}, \psi_{m n}\right\}$ is an orthogonal basis of $H$. Assume throughout this paper that the material parameters $m_{c}, m_{b}, Q$ and $E I$ are chosen such that

$$
\left\{\begin{array}{l}
\text { both } \sqrt{\frac{Q}{m_{c}}} \text { and } \sqrt{\frac{E I}{m_{b}}} \text { are rational numbers } \\
\lambda_{m n}=Q(2 n+1)^{2}-4 m_{c} m^{2} \neq 0 ; \quad \mu_{m n}=E I(2 n+1)^{4}-4 m_{b} m^{2} \neq 0 \\
\lambda_{m n}+\mu_{m n} \neq 0, \text { for } \quad m \geq 1, \quad n \geq 1
\end{array}\right.
$$

By the assumption (2.3), $L_{1}, L_{2}$ and $L_{1}+L_{2}$ are invertible in $H$. The assumption of both $\sqrt{Q / m_{c}}$ and $\sqrt{E I / m_{b}}$ being rational is necessary due to the known fact that certain number theoretical difficulties may be encountered [5]. Define

$$
\mathcal{A}=L_{2} L_{1}\left(L_{1}+L_{2}\right)^{-1} \text {. }
$$

The eigenvalues of $\mathcal{A}$ are given by

$$
\sigma_{m n}=\frac{\lambda_{m n} \mu_{m n}}{\lambda_{m n}+\mu_{m n}}
$$

where the corresponding eigenfunctions are given by $\left\{\varphi_{m n}, \psi_{m n}\right\}$. Under assumption (2.3) and by using (2.2), the following mapping properties of $L_{1}, L_{2}$ and $\mathcal{A}$ were proved in [5].

Lemma 2.1. Let $\beta \in \Re$ and $\beta \neq-\sigma_{m n}$, and $s \geq 0$. Assume that (2.3) holds. Then

(a) $L_{1}^{-1}$ is a bounded linear operator from $H^{s}(\Omega) \cap H$ to $H^{s+1}(\Omega) \cap H$;

(b) $L_{2}^{-1}$ is a bounded linear operator from $H^{s}(\Omega) \cap H$ to $H^{s+2}(\Omega) \cap H$; and

(c) $(\mathcal{A}+\beta)^{-1}$ is a bounded linear operator from $H^{s}(\Omega) \cap H$ to $H^{s+1}(\Omega) \cap H$. 
By Lemma 2.1, it is easy to show that $L_{1}, L_{2}$ and $\mathcal{A}$ have compact inverses in $H$. Under assumption (2.1), one can check easily

$$
\sigma_{20}<\sigma_{10}<0<\sigma_{00}
$$

Assume throughout this paper that

$$
\text { the only eigenvalue of } \mathcal{A} \text { in the interval }\left(\sigma_{20}, \sigma_{00}\right) \text { is } \sigma_{10} \text {. }
$$

By using the above notations and by restricting the domain of $(u, w)$ to $\Omega$, system (1.3) can be written as

$$
\left\{\begin{array}{l}
L_{1} u-K(w-u)^{+}=m_{c} g+\varepsilon h_{1} \\
L_{2} w+K(w-u)^{+}=m_{b} g+\varepsilon h_{2}
\end{array}\right.
$$

By applying the Mountain Pass Theorem to a dual variational formulation of (2.6), it was proved in [5] that if $\left(h_{1}, h_{2}\right) \in\left(H^{2}(\Omega) \cap H\right) \times\left(H^{2}(\Omega) \cap H\right)$, and if $-\sigma_{10}<K<\Delta$ where

$$
-\sigma_{10}<\Delta=\frac{\sigma_{20}+\sqrt{\sigma_{20}^{2}+8 \sigma_{10} \sigma_{20}}}{2}<-\sigma_{20},
$$

then there exists an $\varepsilon_{0}>0$ such that (2.6) admits at least two solutions in $H^{3}(\Omega) \times$ $H^{4}(\Omega)$ if $|\varepsilon|<\varepsilon_{0}$. By relaxing the assumptions on $\left(h_{1}, h_{2}\right)$ and $K$, we prove in this paper the following main result.

Theorem 2.2. Let $\left(h_{1}, h_{2}\right) \in H \times H$ with $\left\|h_{1}\right\|=1$ and $\left\|h_{2}\right\|=1$. If $-\sigma_{10}<K<$ $-\sigma_{20}$, then there exists an $\varepsilon_{0}>0$ such that if $|\varepsilon|<\varepsilon_{0}$, then (2.6) admits at least two solutions in $\left(H^{1}(\Omega) \cap H\right) \times\left(H^{2}(\Omega) \cap H\right)$. Consequently, system (1.3) admits at least two $\pi$-periodic solutions.

In this paper, $\|\cdot\|$ denotes the usual norm of $L^{2}(\Omega)$. To prove the existence of multiple solutions of (2.6), we first derive an equivalent system of (2.6). From (2.6), one has

$$
L_{1} u+L_{2} w=\left(m_{c}+m_{b}\right) g+\varepsilon\left(h_{1}+h_{2}\right) .
$$

By applying $L_{1}^{-1} L_{2}^{-1}$ to both sides of this equation, we have

$$
L_{2}^{-1} u+L_{1}^{-1} w=L_{1}^{-1} L_{2}^{-1}\left[\left(m_{c}+m_{b}\right) g+\varepsilon\left(h_{1}+h_{2}\right)\right] .
$$

Let $\bar{w}=L_{1}^{-1} w$ and $\bar{u}=L_{2}^{-1} u$, then $u=L_{2} \bar{u}, w=L_{1} \bar{w}$, and

$$
\bar{w}+\bar{u}=L_{1}^{-1} L_{2}^{-1}\left[\left(m_{c}+m_{b}\right) g+\varepsilon\left(h_{1}+h_{2}\right)\right] .
$$

By substituting them into the second equation of (2.6), we obtain

$$
L_{2} L_{1} \bar{w}+K\left[\left(L_{1}+L_{2}\right) \bar{w}-\left(m_{c}+m_{b}\right) g L_{1}^{-1}(1)-\varepsilon L_{1}^{-1}\left(h_{1}+h_{2}\right)\right]^{+}=m_{b} g+\varepsilon h_{2} .
$$

Let $v=\left(L_{1}+L_{2}\right) \bar{w}, f_{0}=\left(m_{c}+m_{b}\right) g L_{1}^{-1}(1) \in H$ and $f_{1}=L_{1}^{-1}\left(h_{1}+h_{2}\right) \in H$, then the above equation can be written as

$$
\mathcal{A} v+K\left[v-f_{0}-\varepsilon f_{1}\right]^{+}=m_{b} g+\varepsilon h_{2} .
$$

Note that the relation between $w-u$ and $v$ is given by

$$
w-u=L_{1} \bar{w}-L_{2} \bar{u}=v-f_{0}-\varepsilon f_{1} .
$$


By substituting the above relation into (2.6), we obtain

$$
\left\{\begin{array}{l}
u=L_{1}^{-1}\left[K\left(v-f_{0}-\varepsilon f_{1}\right)^{+}+m_{c} g+\varepsilon h_{1}\right], \\
w=L_{2}^{-1}\left[-K\left(v-f_{0}-\varepsilon f_{1}\right)^{+}+m_{b} g+\varepsilon h_{2}\right] .
\end{array}\right.
$$

If $v \in H$ is a solution of (2.7), then $(u, w) \in\left(H^{1}(\Omega) \cap H\right) \times\left(H^{2}(\Omega) \cap H\right)$ given by (2.9) is a solution of (2.6), where the regularity of $(u, w)$ is obtained by applying Lemma 2.1. Therefore, to study the multiple solutions of (2.6) becomes to study the multiple solutions of (2.7). We prove (2.7) admits at least two solutions in $H$ by using the Leray-Schauder degree theory.

We need to establish several useful lemmas. Consider the equilibrium oscillation in system (1.3) determined by the following equation,

$$
\left\{\begin{array}{l}
-Q u_{x x}-K(w-u)^{+}=m_{c} g, \quad-\pi / 2<x<\pi / 2 \\
E I w_{x x x x}+K(w-u)^{+}=m_{b} g, \quad-\pi / 2<x<\pi / 2, \\
u(-\pi / 2)=u(\pi / 2)=0, \\
w(-\pi / 2)=w(\pi / 2)=0, \quad w_{x x}(-\pi / 2)=w_{x x}(\pi / 2)=0 \\
u(-x)=u(x), \quad w(-x)=w(x), \quad 0 \leq x \leq \pi / 2 .
\end{array}\right.
$$

Lemma 2.3. For any given $K>0$, there exists $a \mu_{0}>0$, which depends only on $K, Q$ and $E I$, such that if $m_{c} / m_{b}<\mu_{0}$, then (2.10) admits a $C^{\infty}$-solution $\left(u_{e}, w_{e}\right)$ satisfying $w_{e}^{\prime}(-\pi / 2)-u_{e}^{\prime}(-\pi / 2)>0, w_{e}^{\prime}(\pi / 2)-u_{e}^{\prime}(\pi / 2)<0$, and $w_{e}(x)-u_{e}(x)>0$ for $-\pi / 2<x<\pi / 2$.

Note that $\left(u_{e}, w_{e}\right)$ obviously satisfies (2.6) with $\varepsilon=0$,

$$
\left\{\begin{array}{l}
L_{1} u_{e}-K\left(w_{e}-u_{e}\right)^{+}=m_{c} g \\
L_{2} w_{e}+K\left(w_{e}-u_{e}\right)^{+}=m_{b} g
\end{array}\right.
$$

The proof of Lemma 2.3 and the explicit expressions of $\mu_{0}$ and $\left(u_{e}, w_{e}\right)$ can be found in [5]. Thus by (2.8), $v_{0}=w_{e}-u_{e}+f_{0} \in C^{\infty}(\Omega) \cap H$ satisfying

$$
\mathcal{A} v_{0}+K\left[v_{0}-f_{0}\right]^{+}=m_{b} g,
$$

and $v_{0}(x, t)-f_{0}(x, t)=w_{e}(x)-u_{e}(x)>0$ for $-\pi / 2<x<\pi / 2$, and $v_{0}( \pm \pi / 2, t)-$ $f_{0}( \pm \pi / 2, t)=0$.

Lemma 2.4. If $-\sigma_{00}<K<-\sigma_{20}$, then the following equation

$$
\mathcal{A} v+K v^{+}=0
$$

admits only the trivial solution $v=0$ in $H$.

The proof of Lemma 2.4 can be found in [5]. The next lemma establishes an $a$ priori bound for solutions of (2.7) in $H$.

Lemma 2.5. Let $\left(h_{1}, h_{2}\right) \in H \times H$ with $\left\|h_{1}\right\|=1$ and $\left\|h_{2}\right\|=1$. Let $\alpha>0$ be a given small real number. Then there exists an $R_{0}>0$ depending only on $\alpha$ and $\left(h_{1}, h_{2}\right)$ such that if $-\sigma_{00}+\alpha \leq K \leq-\sigma_{20}-\alpha$ and $\epsilon \in[-1,1]$, any solution $v$ of (2.7) satisfies $\|v\|<R_{0}$.

Proof. Assume the conclusion is not true, then there exist sequences of $\left\{\varepsilon_{n}\right\},\left\{K_{n}\right\}$ and $\left\{v_{n}\right\}$ such that $K_{n} \in\left[-\sigma_{00}+\alpha,-\sigma_{20}-\alpha\right], \varepsilon_{n} \in[-1,1],\left\|v_{n}\right\| \rightarrow \infty$, and

$$
\mathcal{A} v_{n}+K_{n}\left[v_{n}-f_{0}-\varepsilon_{n} f_{1}\right]^{+}=m_{b} g+\varepsilon_{n} h_{2} \text {. }
$$


Let $\bar{v}_{n}=\frac{v_{n}}{\left\|v_{n}\right\|}$, then

$$
\bar{v}_{n}=\mathcal{A}^{-1}\left\{\frac{m_{b} g}{\left\|v_{n}\right\|}+\varepsilon_{n} \frac{h_{2}}{\left\|v_{n}\right\|}-K_{n}\left[\bar{v}_{n}-\frac{f_{0}}{\left\|v_{n}\right\|}-\varepsilon_{n} \frac{f_{1}}{\left\|v_{n}\right\|}\right]^{+}\right\} .
$$

Since $\mathcal{A}^{-1}$ is compact in $H$, there is a subsequence of $\left\{\bar{v}_{n}\right\}$; denote it again by $\left\{\bar{v}_{n}\right\}$, such that $\bar{v}_{n} \rightarrow \bar{v}_{0}, K_{n} \rightarrow K_{0}$ and $\varepsilon_{n} \rightarrow \varepsilon_{0}$, and

$$
\bar{v}_{0}=\mathcal{A}^{-1}\left\{-K_{0}\left[\bar{v}_{0}\right]^{+}\right\},
$$

where $K_{0} \in\left[-\sigma_{00}+\alpha,-\sigma_{20}-\alpha\right] \subset\left(-\sigma_{00},-\sigma_{20}\right), \varepsilon_{0} \in[-1,1]$ and $\left\|\bar{v}_{0}\right\|=1$. However, by Lemma 2.4 the above equation admits only the trivial solution $\bar{v}_{0}=0$, which contradicts $\left\|\bar{v}_{0}\right\|=1$.

Lemma 2.6. Let $\left(h_{1}, h_{2}\right) \in H \times H$ with $\left\|h_{1}\right\|=1$ and $\left\|h_{2}\right\|=1$. Let $\alpha>0$ be a given small real number, and let $R_{0}$ be defined as in Lemma 2.5. If $K \in$ $\left[-\sigma_{00}+\alpha,-\sigma_{20}-\alpha\right]$ and $\varepsilon \in[-1,1]$, then

$$
d_{L S}\left(v-\mathcal{A}^{-1}\left\{m_{b} g+\varepsilon h_{2}-K\left[v-f_{0}-\varepsilon f_{1}\right]^{+}\right\}, B_{R}(0), 0\right)=1,
$$

for all $R \geq R_{0}$, where $d_{L S}$ denotes the Leray-Schauder degree, and $B_{R}(0)=\{v \in$ $H \mid\|v\| \leq R\}$.

Proof. Let $R \geq R_{0}$. For any $K \in\left[-\sigma_{00}+\alpha,-\sigma_{20}-\alpha\right]$, define

$$
\psi_{K}(v)=\mathcal{A}^{-1}\left\{m_{b} g+\varepsilon h_{2}-K\left[v-f_{0}-\varepsilon f_{1}\right]^{+}\right\} .
$$

From Lemma 2.5, any solution $v$ of (2.7) is bounded and satisfies $\|v\|<R_{0}$. Thus $0 \notin\left(I-\psi_{K}\right)\left(\partial B_{R}(0)\right)$. Since $\mathcal{A}^{-1}$ is compact in $H, \psi_{K}$ defines a homotopy of compact transformation on $B_{R}(0)$. Note that

$$
v-\psi_{0}(v)=v-\mathcal{A}^{-1}\left(m_{b} g+\varepsilon h_{2}\right)
$$

which is simply a translation of the identity, and $\left\|\mathcal{A}^{-1}\left(m_{b} g+\varepsilon h_{2}\right)\right\|<R_{0}$ by Lemma 2.5. Then by using the properties of the Leray-Schauder degree [11, we have

$$
d_{L S}\left(v-\psi_{0}(v), B_{R}(0), 0\right)=1 .
$$

By the invariance of the Leray-Schauder degree under homotopy [1], we have

$$
d_{L S}\left(v-\psi_{K}(v), B_{R}(0), 0\right)=1,
$$

for $K \in\left[-\sigma_{00}+\alpha,-\sigma_{20}-\alpha\right]$.

The next important lemma was first introduced and proved by McKenna and Walter in 13 .

Lemma 2.7. Let $D$ be a compact set in $L^{2}(\Omega)$, and $\phi \in L^{2}(\Omega)$ be positive almost everywhere. Then there exists a modulus of continuity $\delta$ depending only on $D$ and $\phi$ such that

$$
\left\|(\eta \psi-\phi)^{+}\right\| \leq \eta \delta(\eta), \quad \text { for any } \eta>0 \text { and } \psi \in D .
$$

Lemma 2.7 plays an important role in proving the following lemma.

Lemma 2.8. Let $\left(h_{1}, h_{2}\right) \in H \times H$ with $\left\|h_{1}\right\|=1$ and $\left\|h_{2}\right\|=1$. If $-\sigma_{10}<K<$ $-\sigma_{20}$, then there exist $\gamma>0$ and $\varepsilon_{0}>0$ such that

$$
d_{L S}\left(v-\mathcal{A}^{-1}\left\{m_{b} g+\varepsilon h_{2}-K\left[v-f_{0}-\varepsilon f_{1}\right]^{+}\right\}, B_{\gamma}\left(v_{0}\right), 0\right)=-1,
$$

for $|\varepsilon| \leq \varepsilon_{0}$, where $v_{0}$ is defined in [2.12). 
Proof. For any $\lambda \in[0,1]$, define

$$
h_{\lambda}(v)=\mathcal{A}^{-1}\left\{m_{b} g-K\left(v-f_{0}\right)+\lambda\left[\varepsilon\left(h_{2}+K f_{1}\right)-K\left(v-f_{0}-\varepsilon f_{1}\right)^{-}\right]\right\},
$$

where $w^{-}=\max \{-w, 0\}$ and $w=w^{+}-w^{-}$. Then

$$
\begin{aligned}
& h_{0}(v)=\mathcal{A}^{-1}\left\{m_{b} g-K\left(v-f_{0}\right)\right\}, \\
& h_{1}(v)=\mathcal{A}^{-1}\left\{m_{b} g+\varepsilon h_{2}-K\left(v-f_{0}-\varepsilon f_{1}\right)^{+}\right\} .
\end{aligned}
$$

Since $\mathcal{A}^{-1}$ is compact in $H, h_{\lambda}$ defines a homotopy of compact transformation on $B_{\gamma}\left(v_{0}\right)$ in $H$ for any $\gamma>0$. If, for some $\gamma>0, h_{\lambda}$ satisfies

$$
0 \notin\left(I-h_{\lambda}\right)\left(\partial B_{\gamma}\left(v_{0}\right)\right), \quad \forall \lambda \in[0,1]
$$

then, by the invariance of the Leray-Schauder degree under homotopy [11, we have

$$
\begin{aligned}
d_{L S} & \left(v-\mathcal{A}^{-1}\left\{m_{b} g+\varepsilon h_{2}-K\left[v-f_{0}-\varepsilon f_{1}\right]^{+}\right\}, B_{\gamma}\left(v_{0}\right), 0\right) \\
& =d_{L S}\left(v-h_{1}(v), B_{\gamma}\left(v_{0}\right), 0\right) \\
& =d_{L S}\left(v-h_{0}(v), B_{\gamma}\left(v_{0}\right), 0\right) \\
& =d_{L S}\left(v-\mathcal{A}^{-1}\left\{m_{b} g-K\left(v-f_{0}\right)\right\}, B_{\gamma}\left(v_{0}\right), 0\right) .
\end{aligned}
$$

By Lemma 2.3 and (2.12), it is easy to verify that $v_{0}$ is the unique solution of

$$
\mathcal{A} v+K\left[v-f_{0}\right]=m_{b} g,
$$

because $-\sigma_{10}<K<-\sigma_{20}$. Thus

$$
d_{L S}\left(v-\mathcal{A}^{-1}\left\{m_{b} g-K\left(v-f_{0}\right)\right\}, B_{\gamma}\left(v_{0}\right), 0\right)=d_{L S}\left(\left(I+K \mathcal{A}^{-1}\right) v, B_{\gamma}(0), 0\right) .
$$

By (2.3) , it is easy to check that the eigenvalues of $I+K \mathcal{A}^{-1}$ are given by $\rho_{m n}=$ $1+\frac{K}{\sigma_{m n}} \neq 0$ whose corresponding eigenfunctions are given by $\left\{\varphi_{m n}, \psi_{m n}\right\}$. By (2.5) and the assumption $-\sigma_{10}<K<-\sigma_{20}$, we have $\rho_{10}<0$ and $\rho_{m n}>0$ for the rest of the eigenvalues of $I+K \mathcal{A}^{-1}$. Thus, for such a type of linear operators, it is well known [11 that

$$
d_{L S}\left(\left(I+K \mathcal{A}^{-1}\right) v, B_{\gamma}(0), 0\right)=-1 .
$$

Therefore, we have

$$
d_{L S}\left(v-\mathcal{A}^{-1}\left\{m_{b} g+\varepsilon h_{2}-K\left[v-f_{0}-\varepsilon f_{1}\right]^{+}\right\}, B_{\gamma}\left(v_{0}\right), 0\right)=-1,
$$

provided (2.14) is proved to be true.

In the rest of the proof, we show that there exist $\varepsilon_{0}>0$ and $\gamma>0$ such that (2.14) is true when $|\varepsilon| \leq \varepsilon_{0}$.

Under assumptions (2.1) and (2.3), it is straightforward to check that $0<\sigma_{00}<$ $-\sigma_{10}$, hence $\left\|\mathcal{A}^{-1}\right\|=1 / \sigma_{00}$. Let $D$ be the closure of $\mathcal{A}^{-1}\left(B_{1}(0)\right)$. Thus $D$ is compact in $H$. Assume there is a $v \in H$ such that $\left\|v-v_{0}\right\|=\gamma$ and $v-h_{\lambda}(v)=0$, then

$$
\mathcal{A} v-\left\{m_{b} g-K\left(v-f_{0}\right)+\lambda\left[\varepsilon\left(h_{2}+K f_{1}\right)-K\left(v-f_{0}-\varepsilon f_{1}\right)^{-}\right]\right\}=0 .
$$

Let $\phi=v-v_{0}$. Then $\|\phi\|=\gamma$. By using (2.12), it follows from (2.15) that

$$
\mathcal{A} \phi=-K \phi+\lambda\left[\varepsilon\left(h_{2}+K f_{1}\right)-K\left(v_{0}-f_{0}+\phi-\varepsilon f_{1}\right)^{-}\right] .
$$

Since $v_{0}-f_{0} \geq 0$ on $\Omega$, we have

$$
0 \leq\left(v_{0}-f_{0}+\phi-\varepsilon f_{1}\right)^{-} \leq\left(\phi-\varepsilon f_{1}\right)^{-} .
$$


Thus, for any $\lambda \in[0,1]$,

$$
\left\|-K \phi+\lambda\left[\varepsilon\left(h_{2}+K f_{1}\right)-K\left(v_{0}-f_{0}+\phi-\varepsilon f_{1}\right)^{-}\right]\right\| \leq|\varepsilon|\left(1+2 K\left\|f_{1}\right\|\right)+2 K \gamma .
$$

Let $\varepsilon_{1}=\frac{\gamma}{1+2 K\left\|f_{1}\right\|}$. By (2.16) and the above estimate, we then have, for any $|\varepsilon| \leq \varepsilon_{1}$,

$$
\phi \in(1+2 K) \gamma D
$$

Rewrite 2.16 as

$$
\phi+K \mathcal{A}^{-1} \phi=\lambda \mathcal{A}^{-1}\left[\varepsilon\left(h_{2}+K f_{1}\right)-K\left(v_{0}-f_{0}+\phi-\varepsilon f_{1}\right)^{-}\right] .
$$

By (2.5) and the assumption $-\sigma_{10}<K<-\sigma_{20}$, we have

$$
\alpha \stackrel{\text { def }}{=} \inf _{\psi \in H,\|\psi\|=1}\left\|\psi+K \mathcal{A}^{-1} \psi\right\|>0
$$

$\alpha$ depends only on $K$ and $\mathcal{A}$. Since $\|\phi\|=\gamma$, the left-hand side of (2.18) satisfies

$$
\left\|\phi+K \mathcal{A}^{-1} \phi\right\| \geq \gamma \alpha \text {. }
$$

For the right-hand side of (2.18), we obtain

$$
\begin{aligned}
\left\|\lambda \mathcal{A}^{-1}\left[\varepsilon\left(h_{2}+K f_{1}\right)-K\left(v_{0}-f_{0}+\phi-\varepsilon f_{1}\right)^{-}\right]\right\| \\
\leq \frac{|\varepsilon|\left(1+2 K\left\|f_{1}\right\|\right)}{\sigma_{00}}+\frac{K}{\sigma_{00}}\left\|\left[\phi+v_{0}-f_{0}\right]^{-}\right\|,
\end{aligned}
$$

where we have used $0 \leq[u+w]^{-} \leq u^{-}+w^{-}$and $0 \leq w^{-} \leq|w|$. Note that $D$ is compact in $H$, and $\left(v_{0}-f_{0}\right)(x, t)>0$ for $-\pi / 2<x<\pi / 2$ and $\left(v_{0}-f_{0}\right)( \pm \pi / 2, t)=0$ from 2.12). By Lemma 2.7, there exists a modulus of continuity $\delta$ depending only on $D$ and $v_{0}-f_{0}$ such that

$$
\left\|\left[\eta \psi-\left(v_{0}-f_{0}\right)\right]^{+}\right\| \leq \eta \delta(\eta), \quad \text { for any } \eta>0 \text { and } \psi \in D .
$$

Thus, by using 2.17), we have

$$
\left\|\left[\phi+v_{0}-f_{0}\right]^{-}\right\|=\left\|\left[-\phi-\left(v_{0}-f_{0}\right)\right]^{+}\right\| \leq(1+2 K) \gamma \delta((1+2 K) \gamma) .
$$

Then

$$
\begin{aligned}
\left\|\lambda \mathcal{A}^{-1}\left[\varepsilon\left(h_{2}+K f_{1}\right)-K\left(v_{0}-f_{0}+\phi-\varepsilon f_{1}\right)^{-}\right]\right\| \\
\leq \frac{|\varepsilon|\left(1+2 K\left\|f_{1}\right\|\right)}{\sigma_{00}}+\frac{(1+2 K) K \gamma}{\sigma_{00}} \delta((1+2 K) \gamma) .
\end{aligned}
$$

Since $\delta(\eta)$ is a modulus of continuity, one can choose $\gamma$ small enough such that

$$
\frac{(1+2 K) K}{\sigma_{00}} \delta((1+2 K) \gamma)<\frac{\alpha}{4} .
$$

Then we fix $\gamma$, and let

$$
\varepsilon_{0}=\min \left\{\frac{\gamma}{1+2 K\left\|f_{1}\right\|}, \frac{\alpha \gamma \sigma_{00}}{2\left(1+2 K\left\|f_{1}\right\|\right)}\right\} \leq \varepsilon_{1} .
$$

For any $|\varepsilon| \leq \varepsilon_{0}$, we then have

$$
\left\|\lambda \mathcal{A}^{-1}\left[\varepsilon\left(h_{2}+K f_{1}\right)-K\left(v_{0}-f_{0}+\phi-\varepsilon f_{1}\right)^{-}\right]\right\| \leq \frac{3 \alpha \gamma}{4} .
$$


Since the left-hand side of (2.18) satisfies (2.19), and the right-hand side of (2.18) satisfies (2.20) for any $|\varepsilon| \leq \varepsilon_{0}$, there is no such $\phi \in H$ satisfying (2.18). Hence (2.15) has no solution in $H$ if $|\varepsilon| \leq \varepsilon_{0}$. Therefore, (2.14) is proved.

Proof of Theorem 2.2. Since (2.6) is equivalent to (2.7), one only needs to show that (2.7) admits at least two solutions in $H$. For any $-\sigma_{10}<K<-\sigma_{20}$, it follows from Lemma 2.6 that there exists an $R_{0}>0$ such that, for $R \geq R_{0}$,

$$
d_{L S}\left(v-\mathcal{A}^{-1}\left\{m_{b} g+\varepsilon h_{2}-K\left[v-f_{0}-\varepsilon f_{1}\right]^{+}\right\}, B_{R}(0), 0\right)=1 .
$$

By Lemma 2.8 there exist $\gamma>0$ and $\varepsilon_{0}>0$ such that

$$
d_{L S}\left(v-\mathcal{A}^{-1}\left\{m_{b} g+\varepsilon h_{2}-K\left[v-f_{0}-\varepsilon f_{1}\right]^{+}\right\}, B_{\gamma}\left(v_{0}\right), 0\right)=-1,
$$

for $|\varepsilon| \leq \varepsilon_{0}$. By choosing $R \geq R_{0}$ so large that $B_{R}(0) \supset B_{\gamma}\left(v_{0}\right)$, we then have

$$
d_{L S}\left(v-\mathcal{A}^{-1}\left\{m_{b} g+\varepsilon h_{2}-K\left[v-f_{0}-\varepsilon f_{1}\right]^{+}\right\}, B_{R}(0) \backslash B_{\gamma}\left(v_{0}\right), 0\right)=2 .
$$

Therefore, (2.7) admits at least two solutions in $H$, one in $B_{\gamma}\left(v_{0}\right)$ and one in $B_{R}(0) \backslash B_{\gamma}\left(v_{0}\right)$. Consequently, (2.6) admits at least two solutions in $H \times H$.

From the above proof of Theorem [2.2, we observe that one solution $v_{1}$ of (2.7) is in $B_{\gamma}\left(v_{0}\right)$, which is very close to $v_{0}$. In other words, (2.6) admits a solution corresponding to $v_{1}$ by (2.9), which is in fact a near-equilibrium solution. Such a near-equilibrium solution can be proved also by the Banach fixed point theorem [5]. On the other hand, the above proof of Theorem 2.2 shows that (2.7) admits another solution $v_{2}$ in $B_{R}(0) \backslash B_{\gamma}\left(v_{0}\right)$, which implies $\left\|v_{2}-v_{0}\right\|>\gamma$. In other words, (2.6) admits a solution corresponding to $v_{2}$ by (2.9), which is not near the equilibrium solution $\left(u_{e}, w_{e}\right)$. In this sense, such a solution can be understood as a large amplitude oscillation of system (2.6).

As a final remark, we point out that assumption (2.1) plays a key role in proving Lemma 2.4, which plays a key role in establishing a priori bound for solutions of (2.7) in $H$ (see Lemma 2.5), and in proving that the functional corresponding to the variational formulation of system (2.7) satisfies the Palais-Smale condition in [5]. (2.1) is a sufficient condition, and can be relaxed certainly a little bit further. However, a relaxation of (2.1) may create some technical difficulties particularly in proving Lemma 2.4

\section{REFERENCES}

1. O. H. Amann, T. Von Karman and G. B. Woodruff, The failure of the Tacoma Narrows Bridge, Federal Works Agency, Washington D. C., 1941.

2. N. U. Ahmed and H. Harbi, Mathematical analysis of dynamic models of suspension bridges, SIAM J. Appl. Math., 58 (1998), 853-874. MR 99d:73050

3. Q. H. Choi, T. Jung and P. J. McKenna, The study of a nonlinear suspension bridge equation by a variational reduction method, Appl. Anal., 50 (1993), 73-92. MR 95h:35232

4. Z. Ding, Nonlinear periodic oscillations in suspension bridges, in Control of nonlinear distributed systems, ed. by G. Chen, I. Lasiecka and J. Zhou, Marcel Dekker, 2001, pp. 69-84. CMP 2001:09

5. Z. Ding, Nonlinear periodic oscillations in a suspension bridge system under periodic external aerodynamic forces, to appear in Nonlinear Anal.

6. P. Drabek, H. Leinfelder and G. Tajcova, Coupled string-beam equations as a model of suspension bridges, Appl. Math., 44 (1999), 97-142. MR 2000a:74066

7. D. Gilbarg and N. S. Trudinger, Elliptic partial differential equations of second order, Springer, New York, 1983. MR 86c:35035

8. L. D. Humphreys, Numerical mountain pass solutions of a suspension bridge equation, Nonlinear Anal., 28 (1997), 1811-1826. MR 98g:65099 
9. L. D. Humphreys and P. J. McKenna, Multiple periodic solutions for a nonlinear suspension bridge equation, IMA J. Appl. Math., 63 (1999), 37-49. MR 2001e:35162

10. A. C. Lazer and P. J. McKenna, Large-amplitude periodic oscillations in suspension bridges: Some new connections with nonlinear analysis, SIAM Review, 32 (1990), 537-578. MR 92g:73059

11. N. G. Lloyd, Degree Theory, Cambridge University Press, New York, 1978. MR 58:12558

12. P. J. McKenna and W. Walter, Nonlinear oscillations in a suspension bridge, Arch. Rational Mech. Anal., 98 (1987), 167-177. MR 88a:35160

13. P. J. McKenna and W. Walter, On the multiplicity of the solution set of some nonlinear boundary value problems, Nonlinear Anal., 8 (1984), 893-907. MR 85j:35074

14. G. Tajcova, Mathematical models of suspension bridges, Appl. Math., 42 (1997), 451-480. MR 98k:73047

Department of Mathematical Sciences, University of Nevada, Las Vegas, Nevada $89154-4020$

E-mail address: dingz@nevada.edu 